\title{
Strong convergence theorems for the split equality variational inclusion problem and fixed point problem in Hilbert spaces
}

\author{
Haili Guo' ${ }^{1}$ Huimin $\mathrm{He}^{2}$ and Rudong Chen ${ }^{1 *}$
}

"Correspondence:

chenrd@tjpu.edu.cn

'Department of Mathematics,

Tianjin Polytechnic University,

Tianjin, 300387, China

Full list of author information is

available at the end of the article

\begin{abstract}
In this paper, we propose and investigate two new iterative algorithms for solving the split equality variational inclusion problem in Hilbert spaces. We also prove that the sequences generated by the proposed algorithms converge strongly to a common solution of the split equality variational inclusion problem and fixed points of a family of nonexpansive mappings, which is also an unique solution of a variational inequality as an optimality condition for a minimization problem. The results presented in this paper extend and generalize a variety of existing results in this area.
\end{abstract}

Keywords: split equality problem; split equality variational inclusion problem; fixed point; variational inequality

\section{Introduction}

In this paper, we consider the split equality problem (SEP) proposed by Moudafi [1]. Let $H_{1}$, $H_{2}, H_{3}$ be real Hilbert spaces, $S_{n}: H_{1} \rightarrow H_{1}$ be a family of nonexpansive mappings, $\operatorname{Fix}\left(S_{n}\right)$ denote the fixed points set of $S_{n}, n=1,2, \ldots, C=\bigcap_{n=1}^{\infty} \operatorname{Fix}\left(S_{n}\right) \in H_{1}, Q$ be the nonempty closed convex set of $H_{2}$. Let $A: H_{1} \rightarrow H_{3}, B: H_{2} \rightarrow H_{3}$ be two bounded linear operators. The so-called SEP can mathematically be formulated as finding $x \in C, y \in Q$ satisfying the property:

$$
x \in C, y \in Q, \quad A x=B y .
$$

Throughout this paper, we use $\Gamma$ to denote the solution set of SEP, that is,

$$
\Gamma=\left\{(x, y) \in H_{1} \times H_{2}, A x=B y, x \in C, y \in Q\right\} .
$$

If $B=I$ (the identity mapping on Hilbert space $H$ ), the problem (1) is equivalent to the well-known split feasibility problem (SFP). It is easy to see that the SEP (1) includes the SFP as a special case. The split equality problems allow asymmetric and partial relations between the variables $x$ and $y$. As is well known, the SEP has received much attention due to its application in various disciplines such as medical image reconstruction, game theory, decomposition methods for PDEs, and radiation therapy treatment planning [2-4].

\section{Springer}


In 2011, Moudafi [5] introduced and studied the following split variational inclusion problem (SVIP). Let $H_{1}, H_{2}$ be Hilbert spaces, $A: H_{1} \rightarrow H_{2}$ be a bounded linear operator, $A^{*}$ be the adjoint of $A$, and $B_{1}: H_{1} \rightarrow H_{1}, B_{2}: H_{2} \rightarrow H_{2}$ be two set-valued maximal monotone mappings. SVIP is formulated as the following problem:

$$
\text { find } x^{*} \in H_{1} \text { such that } 0 \in B_{1}\left(x^{*}\right), 0 \in B_{2}\left(A x^{*}\right) \text {. }
$$

Recently, Byrne et al. [6] proposed the following iterative method to solve the problem (2): For given $x_{0} \in H_{1}$ and $\lambda>0$, the iterative sequence $\left\{x_{n}\right\}$ is generated as follows:

$$
x_{n+1}=J_{\lambda}^{B_{1}}\left[x_{n}+\gamma A^{*}\left(J_{\lambda}^{B_{2}}-I\right) A x_{n}\right]
$$

Moreover, iterative methods for nonexpansive mappings have been applied to solve minimization problem. Moudafi [7] proposed the viscosity approximation method: For every initial $x_{0} \in H$, the sequence $\left\{x_{n}\right\}$ is generated by

$$
x_{n+1}=\alpha_{n} f\left(x_{n}\right)+\left(1-\alpha_{n}\right) T x_{n}
$$

under some certain appropriate conditions imposed on $\left\{\alpha_{n}\right\}$, and it is proved that the sequence generated by (4) converges strongly to the unique solution $x^{*}$ of the variational inequality

$$
\left\langle(I-f) x^{*}, x-x^{*}\right\rangle \geq 0, \quad x \in C
$$

For the iterative method (4), Marino and $\mathrm{Xu}[8]$ introduced a new viscosity approximation method and considered the following iterative sequence $\left\{x_{n}\right\}$ :

$$
x_{n+1}=\alpha_{n} \gamma f\left(x_{n}\right)+\left(I-\alpha_{n} A\right) T x_{n},
$$

and they proved that the sequence generated by (5) converges strongly to the unique solution $x^{*}$ of the variational inequality

$$
\left\langle(A-\gamma f) x^{*}, x-x^{*}\right\rangle \geq 0, \quad x \in C
$$

which is the optimality condition for the following minimization problem:

$$
\min \frac{1}{2}\langle A x, x\rangle-h(x)
$$

where $h$ is a potential function for $\gamma f$.

In 2013, Kazmi and Rizvi [9] combined the iterative method (3) and the viscosity approximation method (4) for solving a split variational inclusion and the fixed point problem of a nonexpansive mapping. Kazmi and Rizvi presented the following iteration scheme:

$$
\left\{\begin{array}{l}
u_{n}=J_{\lambda}^{B_{1}}\left[x_{n}+\gamma A^{*}\left(J_{\lambda}^{B_{2}}-I\right) A x_{n}\right] \\
x_{n+1}=\alpha_{n} f\left(x_{n}\right)+\left(1-\alpha_{n}\right) T u_{n},
\end{array}\right.
$$


and they proved that the sequences $\left\{u_{n}\right\},\left\{x_{n}\right\}$ converge strongly to $z \in \operatorname{Fix}(T) \cap \Gamma$, where $z=P_{\mathrm{Fix}(T) \cap \Gamma} f(z), \Gamma$ is the solution set of SVIP.

In 2015, Sitthithakerngkiet et al. [10] combined the iterative method (3) and the viscosity approximation method (5) for solving a split variational inclusion and the fixed point problem of a family of nonexpansive mappings. They proposed the following iteration algorithm:

$$
\left\{\begin{array}{l}
y_{n}=J_{\lambda}^{B_{1}}\left[x_{n}+\gamma A^{*}\left(J_{\lambda}^{B_{2}}-I\right) A x_{n}\right] \\
x_{n+1}=\alpha_{n} \xi f\left(x_{n}\right)+\left(I-\alpha_{n} D\right) S_{n} y_{n},
\end{array}\right.
$$

and they proved that the sequence converges strongly to a common solution of SVIP and the fixed point of a family of nonexpansive mappings.

Inspired and motivated by the corresponding convergence results of (1), (2), and (7), we consider the split equality variational inclusion problem (SEVIP):

$$
\text { find } x \in U^{-1}(0)=\operatorname{Fix}\left(J_{u_{n}}^{U}\right), y \in K^{-1}(0)=\operatorname{Fix}\left(J_{u_{n}}^{K}\right) \text { such that } A x=B y \text {, }
$$

where $H_{1}, H_{2}, H_{3}$ are real Hilbert spaces, $U: H_{1} \rightarrow 2^{H_{1}}$ and $K: H_{2} \rightarrow 2^{H_{2}}$ are set-valued maximal monotone mappings, $A: H_{1} \rightarrow H_{3}, B: H_{2} \rightarrow H_{3}$ are two bounded linear operators.

In this paper, we will introduce a more general iterative method for SEVIP (8) and a fixed point problem, which is defined in the following way:

$$
\left\{\begin{array}{l}
v_{n}=J_{u_{n}}^{(U, K)}\left(I-\gamma G^{*} G\right) w_{n}, \\
w_{n}=\alpha_{n} \sigma f\left(w_{n}\right)+\left(I-\alpha_{n} D\right) S_{n} v_{n},
\end{array}\right.
$$

where $\sigma \in[0,1], \alpha_{n} \in(0,1)$, and $D$ is a strongly positive bounded linear operator. Note that, if $\sigma=1, u_{n}=\lambda, D=I, B=I, S_{n}=T$, scheme (9) can be reduced to (7), that is, the iterative method (9) for solving the split equality variational inclusion problem can be reduced to the iterative method (7) for solving SVIP and SFP.

Meanwhile, we will prove that the sequences generated by (9) converge strongly to a common element of the solution set of a split equality variational inclusion problem and the common fixed point set of a family of nonexpansive mappings, which is also an unique solution of a variational inequality as an optimality condition for a minimization problem.

\section{Preliminaries}

We first recall that some definitions, notations, and conclusions which will be used in the proofs of our main results. Let $H$ be a real Hilbert space with inner product $\langle\cdot, \cdot\rangle$ and the norm $\|\cdot\|$. We denote by ' $\rightarrow$ ' strong convergence, by ' $\rightarrow$ ' weak convergence. In order to establish our convergence theorems, we need the following concepts.

\section{Definition 2.1}

(1) A mapping $f: H \rightarrow H$ is $k$-contractive if there exists a constant $k \in(0,1)$ such that

$$
\|f x-f y\| \leq k\|x-y\|, \quad \forall x, y \in H .
$$


(2) A mapping $T$ is nonexpansive if

$$
\|T x-T y\| \leq\|x-y\|, \quad \forall x, y \in H
$$

(3) A mapping $T$ is monotone if

$$
\langle T x-T y, x-y\rangle \geq 0, \quad \forall x, y \in H .
$$

(4) A mapping $T$ is firmly nonexpansive if

$$
\|T x-T y\|^{2} \leq\langle x-y, T x-T y\rangle, \quad \forall x, y \in H .
$$

(5) A bounded linear operator $D$ is said to be strongly positive if there exists a constant $\alpha>0$ such that

$$
\langle D x, x\rangle \geq \alpha\|x\|^{2}, \quad \forall x \in H .
$$

(6) A mapping $P_{C}$ is called the metric projection of $H$ onto $C$, if $P_{C} x$ is the unique point in $C$ with the property

$$
\left\|x-P_{C} x\right\|=\min \{\|x-y\|: y \in C\}, \quad \forall x \in H .
$$

Moreover, $P_{C}$ is characterized by the following properties:

$$
\left\langle x-P_{C} x, y-P_{C} x\right\rangle \leq 0, \quad \forall y \in C .
$$

Proposition 2.1 A Banach space $E$ is said to have the Opial property, if for any sequence $\left\{x_{n}\right\}$ with $x_{n} \rightarrow x^{*}$, we have

$$
\liminf _{n \rightarrow \infty}\left\|x_{n}-x^{*}\right\|<\liminf _{n \rightarrow \infty}\left\|x_{n}-y\right\|
$$

$\forall y \in E$ with $y \neq x^{*}$.

Proposition 2.2 In Hilbert spaces, the following inequalities hold:

$$
\begin{aligned}
& \|x+y\|^{2} \leq\|x\|^{2}+2\langle y, x+y\rangle, \quad \forall x, y \in H, \\
& \langle x, y\rangle=\frac{1}{2}\left(\|x\|^{2}+\|y\|^{2}-\|x-y\|^{2}\right), \quad \forall x, y \in H .
\end{aligned}
$$

Lemma 2.1 ([11]) Assume D is a strongly positive linear bounded operator on a Hilbert $H$ with coefficient $\bar{\gamma}>0$ and $0<\alpha \leq\|D\|^{-1}$, then $\|I-\alpha D\| \leq 1-\alpha \bar{\gamma}$.

Lemma 2.2 ([11]) Let $C$ be a nonempty, closed, and convex subset of a Hilbert space $H$. Assume that $f: C \rightarrow C$ is a contraction with a coefficient $k \in(0,1)$ and $D$ is a strongly positive linear bounded operator with a coefficient $\bar{\gamma}>0$. Then, for $0<\gamma<\frac{\bar{\gamma}}{k}$,

$$
\langle x-y,(D-\gamma f) x-(D-\gamma f) y\rangle \geq(\bar{\gamma}-\gamma k)\|x-y\|^{2}, \quad \forall x, y \in H
$$

That is, $D-\gamma f$ is strongly monotone with coefficient $\bar{\gamma}-\gamma k$. 
Lemma 2.3 ([12]) Let C be a nonempty closed subset of a real Hilbert space $H$, and let $\left\{S_{n}\right\}$ be a sequence of mappings from C into itself. Suppose that $\left\{S_{n}\right\}$ satisfies the AKTT condition: $\sum_{n=1}^{n=\infty} \sup \left\{\left\|S_{n+1} v-S_{n} v\right\|: v \in C\right\}<\infty$. Then for each $x \in C,\left\{S_{n} x\right\}$ converges strongly to a point in C. Furthermore, let $S: C \rightarrow C$ be defined by

$$
S x=S_{n} x, \quad \forall x \in C .
$$

Then $\lim _{n \rightarrow \infty} \sup \left\{\left\|S v-S_{n} v\right\|: v \in C\right\}=0$.

Lemma 2.4 ([13]) Assume $a_{n}$ is a sequence of nonnegative numbers such that $a_{n+1} \leq(1-$ $\left.\gamma_{n}\right) a_{n}+\delta_{n}$ where $\left\{\gamma_{n}\right\}$ is a sequence in $(0,1)$ and $\left\{\delta_{n}\right\}$ is a sequence such that:

(i) $\sum_{n=1}^{\infty} \gamma_{n}=\infty$;

(ii) $\lim \sup _{n \rightarrow \infty} \frac{\delta_{n}}{\gamma_{n}} \leq 0$ or $\sum_{n=1}^{\infty}\left|\delta_{n}\right|<\infty$.

Then $\lim _{n \rightarrow \infty} a_{n}=0$.

Lemma 2.5 ([14]) Let $U$ be a set-valued maximal monotone operator on $H$. For $u>0$, we define the resolvent $J_{u}^{U}=(I+u U)^{-1}$, then the following holds:

(i) For each $u>0, J_{u}^{U}$ is a single-valued and firmly nonexpansive mapping.

(ii) $D\left(J_{u}^{U}\right)=H$ and $\operatorname{Fix}\left(J_{u}^{U}\right)=U^{-1}(0)=\{x \in D(G): 0 \in U x\}$.

(iii) $\left\|J_{\alpha}^{U} x-J_{\beta}^{U} x\right\|^{2} \leq \frac{\alpha-\beta}{\alpha}\left\langle J_{\alpha}^{U} x-J_{\beta}^{U} x, J_{\alpha}^{U} x-x\right\rangle$, for all $\alpha, \beta>0$ and $x \in H$.

In fact $\left\|J_{\alpha}^{U} x-J_{\beta}^{U} x\right\| \leq \frac{|\alpha-\beta|}{\alpha}\left\|J_{\alpha}^{U} x-x\right\|$.

(iv) Suppose that $U^{-1}(0) \neq \emptyset$, then $\left\langle x-J_{\alpha}^{U} x, J_{\alpha}^{U} x-w\right\rangle \geq 0$ for each $x \in H$ and each $w \in U^{-1}(0)$, and each $\beta>0$.

Lemma 2.6 ([15]) Let $\left\{S_{n}\right\}$ be a sequence of real numbers that does not decrease at infinity, in the sense that there exists a subsequence $\left\{S_{n_{j}}\right\}_{j \geq 0}$ of $\left\{S_{n}\right\}$ such that

$$
\left\{S_{n_{j}}\right\}<\left\{S_{n_{j}+1}\right\} \quad \text { for all } j \geq 0 .
$$

Also consider the sequence of the integers $\{\tau(n)\}_{n \geq n_{0}}$ defined by

$$
\tau(n)=\max \left\{k \leq n \mid S_{k}<S_{k+1}\right\} .
$$

Then $\{\tau(n)\}_{n \geq n_{0}}$ is a nondecreasing sequence verifying $\lim _{n \rightarrow \infty} \tau(n)=\infty$, and for all $n \geq n_{0}$, the following two estimates hold:

$$
S_{\tau(n)} \leq S_{\tau(n)+1}, \quad S_{n} \leq S_{\tau(n)+1} .
$$

\section{Main result}

In this section, the following supposed conditions always hold:

(1) Let $H_{1}, H_{2}, H_{3}$ be Hilbert spaces.

(2) Let $U$ and $K$ be two set-valued maximal monotone mappings.

(3) Let $A: H_{1} \rightarrow H_{3}, B: H_{2} \rightarrow H_{3}$ be two bounded linear operators and $A^{*}, B^{*}$ be the adjoint of $A$ and $B$.

(4) $f=\left[\begin{array}{l}f_{1} \\ f_{2}\end{array}\right]$, where $f_{i}, i=1,2$ is a contraction mapping on $H_{i}$ with constant $k \in(0,1)$.

(5) Let $S_{n}$ be a sequence of nonexpansive mappings on $H_{1}, D$ be a strongly positive bounded linear operator with coefficient $\bar{\gamma}>0$. 
(6) Assume the solution set of SEVIP (3) $\Gamma \neq \emptyset$,

$$
J_{u_{n}}^{(U, K)}=\left[\begin{array}{c}
J_{u_{n}}^{U} \\
J_{u_{n}}^{K}
\end{array}\right], \quad G=\left[\begin{array}{ll}
A & -B
\end{array}\right], \quad G^{*} G=\left[\begin{array}{cc}
A^{*} A & -A^{*} B \\
-B^{*} A & B^{*} B
\end{array}\right] .
$$

Proposition 3.1 Let $T=I-\gamma G^{*} G: H_{1} \times H_{2} \longrightarrow H_{1} \times H_{2}$, where $\gamma \in\left(0, \frac{2}{L}\right)$, with $L=$ $\rho\left(G^{*} G\right)$ being the spectral radius of the self adjoint operator $G^{*} G$ on $H_{1} \times H_{2}$, then $T$ is a nonexpansive mapping.

Proof In fact, for any $x, y \in H_{1} \times H_{2}$,

$$
\begin{aligned}
\|T x-T y\|^{2} & =\left\|\left(I-\gamma G^{*} G\right) x-\left(I-\gamma G^{*} G\right) y\right\|^{2} \\
& =\left\|x-y-\gamma G^{*} G(x-y)\right\|^{2} \\
& =\|x-y\|^{2}+\gamma^{2}\left\|G^{*} G(x-y)\right\|^{2}-2 \gamma\left\langle x-y, G^{*} G(x-y)\right\rangle \\
& \leq\|x-y\|^{2}+\gamma^{2} L\|G(x-y)\|^{2}-2 \gamma\langle G(x-y), G(x-y)\rangle \\
& =\|x-y\|^{2}+\gamma^{2} L\|G(x-y)\|^{2}-2 \gamma\|G(x-y)\|^{2} \\
& =\|x-y\|^{2}-\gamma(2-\gamma L)\|G(x-y)\|^{2} \\
& \leq\|x-y\|^{2} .
\end{aligned}
$$

This completes the proof of Proposition 3.1.

Lemma 3.1 ([13]) Let $H_{1}, H_{2}, H_{3}, A, B, A^{*}, B^{*}, U, K, J_{u_{n}}^{(U, K)}, G, G^{*}, f, S_{n}, D$, $S$ be the same as above. If $\Gamma \neq \emptyset$ (the solution set of SEVIP (8)), then $w^{*}=\left(x^{*}, y^{*}\right) \in H_{1} \times H_{2}$ is a solution of SEVIP (8) if and only iffor any given $\gamma>0$ and $u>0$

$$
w^{*}=J_{u}^{(U, K)}\left(I-\gamma G^{*} G\right) w^{*}
$$

Theorem 3.1 Let $H_{1}, H_{2}, H_{3}, A, B, A^{*}, B^{*}, U, K, J_{u_{n}}^{(U, K)}, G, G^{*}, f, S_{n}, D, S$ be the same as above. Let $w_{n}$ be generated by

$$
\left\{\begin{array}{l}
v_{n}=J_{u_{n}}^{(U, K)}\left(I-\gamma G^{*} G\right) w_{n} \\
w_{n}=\alpha_{n} \sigma f\left(w_{n}\right)+\left(I-\alpha_{n} D\right) S_{n} v_{n}
\end{array}\right.
$$

Suppose $S_{n}$ satisfies the AKTT condition, $\operatorname{Fix}(S)=\bigcap_{i=1}^{\infty} \operatorname{Fix}\left(S_{n}\right)$.

If the solution set $\Omega=\operatorname{Fix}(S) \cap \Gamma$ is nonempty and the following conditions are satisfied:

(i) $\alpha_{n} \in(0,1), \lim _{n \rightarrow \infty} \alpha_{n}=0$;

(ii) $0<\bar{\gamma}<\frac{1}{\alpha_{n}}, 0<\sigma<\frac{\bar{\gamma}}{k}$.

Then the sequence $w_{n}$ converges strongly to a point $w^{*}$, where $w^{*}=P_{\Omega}(I-D-\sigma f)\left(w^{*}\right)$ is a unique solution of the variational inequalities

$$
\left\langle(D-\sigma f) w^{*}, w^{*}-z\right\rangle \leq 0, \quad z \in \Omega .
$$

Proof First, we show that $w_{n}$ defined by (12) is well defined. 
We define a mapping

$$
W_{n}=\alpha_{n} \sigma f\left(w_{n}\right)+\left(I-\alpha_{n} D\right) S_{n} J_{u_{n}}^{(U, K)}\left(I-\gamma G^{*} G\right) w_{n}, \quad n \geq 0
$$

By Lemma 2.1, Proposition 3.1, and (14), for any $x, y \subseteq H_{1}$, we have

$$
\begin{aligned}
& \left\|W_{n}(x)-W_{n}(y)\right\| \\
& \quad=\left\|\alpha_{n} \sigma f(x)-\alpha_{n} \sigma f(y)+\left(I-\alpha_{n} D\right)\left(S_{n} J_{u_{n}}^{(U, K)}\left(I-\gamma G^{*} G\right) x-S_{n} J_{u_{n}}^{(U, K)}\left(I-\gamma G^{*} G\right) y\right)\right\| \\
& \quad \leq \alpha_{n} \sigma\|f(x)-f(y)\|+\left\|I-\alpha_{n} D\right\|\left\|S_{n} J_{u_{n}}^{(U, K)}\left(I-\gamma G^{*} G\right) x-S_{n} J_{u_{n}}^{(U, K)}\left(I-\gamma G^{*} G\right) y\right\| \\
& \quad \leq \alpha_{n} \sigma k\|x-y\|+\left(1-\alpha_{n} \bar{\gamma}\right)\|x-y\| \\
& \quad=\left(1-\alpha_{n}(\bar{\gamma}-\sigma k)\right)\|x-y\| .
\end{aligned}
$$

Since $0<1-\alpha_{n}(\bar{\gamma}-\sigma k)<1$, it follows that $W_{n}$ is a contraction. Therefore, by the Banach contraction principle, $W_{n}$ has a unique fixed point in $H_{1}$, denoted by $w_{n}$, that is,

$$
w_{n}=\alpha_{n} \sigma f\left(w_{n}\right)+\left(I-\alpha_{n} D\right) S_{n} J_{u_{n}}^{(U, K)}\left(I-\gamma G^{*} G\right) w_{n}
$$

which is exactly (12).

Second, we claim that $w_{n}$ is bounded.

Indeed, take any $z \in \Omega$, we have $z=J_{u_{n}}^{(U, K)}\left(I-\gamma G^{*} G\right) z$ and $z \in \operatorname{Fix}(S)=\bigcap_{n=1}^{\infty} \operatorname{Fix}\left(S_{n}\right)$,

$$
\begin{aligned}
\left\|v_{n}-z\right\| & =\left\|J_{u_{n}}^{(U, K)}\left(I-\gamma G^{*} G\right) w_{n}-z\right\| \\
& =\left\|J_{u_{n}}^{(U, K)}\left(I-\gamma G^{*} G\right) w_{n}-J_{u_{n}}^{(U, K)}\left(I-\gamma G^{*} G\right) z\right\| \\
& \leq\left\|w_{n}-z\right\| .
\end{aligned}
$$

Thus, we derive that

$$
\begin{aligned}
\left\|w_{n}-z\right\| & =\left\|\alpha_{n} \sigma f\left(w_{n}\right)+\left(I-\alpha_{n} D\right) S_{n} v_{n}-z\right\| \\
& =\left\|\alpha_{n} \sigma f\left(w_{n}\right)-\alpha_{n} D z+\left(I-\alpha_{n} D\right) S_{n} v_{n}-\left(I-\alpha_{n} D\right) S_{n} z\right\| \\
& \leq \alpha_{n}\left\|\sigma f\left(w_{n}\right)-D z\right\|+\left\|I-\alpha_{n} D\right\|\left\|v_{n}-z\right\| \\
& \leq \alpha_{n}\left\|\sigma f\left(w_{n}\right)-D z\right\|+\left(1-\alpha_{n} \bar{\gamma}\right)\left\|w_{n}-z\right\| \\
& \leq \alpha_{n}\left\|\sigma\left(f\left(w_{n}\right)-f(z)\right)+(\sigma f(z)-D z)\right\|+\left(1-\alpha_{n} \bar{\gamma}\right)\left\|w_{n}-z\right\| \\
& \leq \alpha_{n} \sigma k\left\|w_{n}-z\right\|+\alpha_{n}\|\sigma f(z)-D z\|+\left(1-\alpha_{n} \bar{\gamma}\right)\left\|w_{n}-z\right\| \\
& =\left(1-\alpha_{n}(\bar{\gamma}-\sigma k)\right)\left\|w_{n}-z\right\|+\alpha_{n}(\bar{\gamma}-\sigma k) \frac{\|\sigma f(z)-D z\|}{\bar{\gamma}-\sigma k} .
\end{aligned}
$$

It follows that

$$
\begin{aligned}
& \alpha_{n}(\bar{\gamma}-\sigma k)\left\|w_{n}-z\right\| \leq \alpha_{n}(\bar{\gamma}-\sigma k) \frac{\|\sigma f(z)-D z\|}{\bar{\gamma}-\sigma k}, \\
& \left\|w_{n}-z\right\| \leq \frac{\|\sigma f(z)-D z\|}{\bar{\gamma}-\sigma k} .
\end{aligned}
$$


Hence the sequence $\left\{w_{n}\right\}$ of $(15)$ is bounded, so are $\left\{v_{n}\right\},\left\{f\left(w_{n}\right)\right\}$, and $\left\{S_{n} v_{n}\right\}$.

Third, we show that $\left\|w_{n}-v_{n}\right\| \rightarrow 0$.

Indeed, for any $z \in \Omega$, we have

$$
\begin{aligned}
\left\|v_{n}-z\right\|^{2} & =\left\|J_{u_{n}}^{(U, K)}\left(I-\gamma G^{*} G\right) w_{n}-z\right\|^{2} \\
& \leq\left\|w_{n}-z-\gamma G^{*} G w_{n}\right\|^{2} \\
& =\left\|w_{n}-z\right\|^{2}+\gamma^{2}\left\|G^{*} G w_{n}\right\|^{2}-2 \gamma\left\langle w_{n}-z, G^{*} G w_{n}\right\rangle \\
& \leq\left\|w_{n}-z\right\|^{2}+\gamma^{2} L\left\|G w_{n}\right\|^{2}-2 \gamma\left\|G w_{n}\right\|^{2} \\
& =\left\|w_{n}-z\right\|^{2}-\gamma(2-\gamma L)\left\|G w_{n}\right\|^{2} .
\end{aligned}
$$

It follows from (10) and (17) that

$$
\begin{aligned}
\left\|w_{n}-z\right\|^{2}= & \left\|\alpha_{n} \sigma f\left(w_{n}\right)+\left(I-\alpha_{n} D\right) S_{n} v_{n}-z\right\|^{2} \\
= & \left\|\left(I-\alpha_{n} D\right)\left(S_{n} v_{n}-z\right)+\alpha_{n}\left(\sigma f\left(w_{n}\right)-D z\right)\right\|^{2} \\
\leq & \left\|\left(I-\alpha_{n} D\right)\left(S_{n} v_{n}-z\right)\right\|^{2}+2 \alpha_{n}\left(\sigma f\left(w_{n}\right)-D z, w_{n}-z\right\rangle \\
\leq & \left(1-\alpha_{n} \bar{\gamma}\right)^{2}\left\|v_{n}-z\right\|^{2}+2 \alpha_{n}\left(\sigma f\left(w_{n}\right)-D z, w_{n}-z\right\rangle \\
\leq & \left(1-\alpha_{n} \bar{\gamma}\right)\left\|w_{n}-z\right\|^{2}-\left(1-\alpha_{n} \bar{\gamma}\right) \gamma(2-\gamma L)\left\|G w_{n}\right\|^{2} \\
& +2 \alpha_{n}\left\|\sigma f\left(w_{n}\right)-D z\right\|\left\|w_{n}-z\right\| .
\end{aligned}
$$

This implies that

$$
\begin{aligned}
\left(1-\alpha_{n} \bar{\gamma}\right) \gamma(2-\gamma L)\left\|G w_{n}\right\|^{2} & \leq-\alpha_{n} \bar{\gamma}\left\|w_{n}-z\right\|^{2}+2 \alpha_{n}\left\|\sigma f\left(w_{n}\right)-D z\right\|\left\|w_{n}-z\right\| \\
& \leq 2 \alpha_{n}\left\|\sigma f\left(w_{n}\right)-D z\right\|\left\|w_{n}-z\right\| .
\end{aligned}
$$

Since both $w_{n}$ and $f\left(w_{n}\right)$ are bounded and $\alpha_{n} \rightarrow 0$, we have $\left\|G w_{n}\right\| \rightarrow 0$.

Then from (11) and Lemma 2.5, we derive that

$$
\begin{aligned}
\left\|v_{n}-z\right\|^{2}= & \left\|J_{u_{i}}^{\left(U_{i}, K_{i}\right)}\left(I-\gamma G^{*} G\right) w_{n}-z\right\|^{2} \\
\leq & \left\langle v_{n}-z, w_{n}-\gamma G^{*} G w_{n}-z\right\rangle \\
= & \frac{1}{2}\left\{\left\|v_{n}-z\right\|^{2}+\left\|w_{n}-z\right\|^{2}-\gamma(2-\gamma L)\left\|G w_{n}\right\|^{2}-\left\|w_{n}-v_{n}\right\|^{2}\right. \\
& \left.-\left\|\gamma G^{*} G w_{n}\right\|^{2}+2\left\langle w_{n}-v_{n}, \gamma G^{*} G w_{n}\right\rangle\right\} .
\end{aligned}
$$

This implies that

$$
\left\|v_{n}-z\right\|^{2} \leq\left\|w_{n}-z\right\|^{2}-\left\|w_{n}-v_{n}\right\|^{2}+2 \gamma \sqrt{L}\left\|w_{n}-v_{n}\right\|\left\|G w_{n}\right\| .
$$

By (18) and (19), we have

$$
\begin{aligned}
\left\|w_{n}-z\right\|^{2} & \leq\left(1-\alpha_{n} \bar{\gamma}\right)^{2}\left\|v_{n}-z\right\|^{2}+2 \alpha_{n}\left\langle\sigma f\left(w_{n}\right)-D z, w_{n}-z\right\rangle \\
& \leq\left(1-\alpha_{n} \bar{\gamma}\right)\left\|v_{n}-z\right\|^{2}+2 \alpha_{n}\left\|\sigma f\left(w_{n}\right)-D z\right\|\left\|w_{n}-z\right\|
\end{aligned}
$$




$$
\begin{aligned}
\leq & \left(1-\alpha_{n} \bar{\gamma}\right)\left\|w_{n}-z\right\|^{2}-\left(1-\alpha_{n} \bar{\gamma}\right)\left\|w_{n}-v_{n}\right\|^{2} \\
& +2\left(1-\alpha_{n} \bar{\gamma}\right) \gamma \sqrt{L}\left\|w_{n}-v_{n}\right\|\left\|G w_{n}\right\|+2 \alpha_{n}\left\|\sigma f\left(w_{n}\right)-D z\right\|\left\|w_{n}-z\right\|
\end{aligned}
$$

Hence, we obtain

$$
\begin{aligned}
\left(1-\alpha_{n} \bar{\gamma}\right)\left\|w_{n}-v_{n}\right\|^{2} \leq & 2\left(1-\alpha_{n} \bar{\gamma}\right) \gamma \sqrt{L}\left\|w_{n}-v_{n}\right\|\left\|G w_{n}\right\| \\
& +2 \alpha_{n}\left\|\sigma f\left(w_{n}\right)-D z\right\|\left\|w_{n}-z\right\|
\end{aligned}
$$

Since $\alpha_{n} \rightarrow 0,\left\|G w_{n}\right\| \rightarrow 0$, it follows that $\left\|w_{n}-v_{n}\right\| \rightarrow 0$.

Nextly, we show $\left\|S v_{n}-v_{n}\right\| \rightarrow 0$.

$$
\begin{aligned}
\left\|w_{n}-S_{n} w_{n}\right\| & =\left\|w_{n}-S_{n} v_{n}+S_{n} v_{n}-S_{n} w_{n}\right\| \\
& \leq\left\|w_{n}-S_{n} v_{n}\right\|+\left\|v_{n}-w_{n}\right\| \\
& =\left\|\alpha_{n} \sigma f\left(w_{n}\right)+\left(I-\alpha_{n} D\right) S_{n} v_{n}-S_{n} v_{n}\right\|+\left\|v_{n}-w_{n}\right\| \\
& =\alpha_{n}\left\|f\left(w_{n}\right)-D S_{n} v_{n}\right\|+\left\|v_{n}-w_{n}\right\| .
\end{aligned}
$$

Since $\left\{f\left(w_{n}\right)\right\}$ and $\left\{S_{n} v_{n}\right\}$ are bounded, $\alpha_{n} \rightarrow 0,\left\|w_{n}-v_{n}\right\| \rightarrow 0$, then $\left\|w_{n}-S_{n} w_{n}\right\| \rightarrow 0$.

Thus,

$$
\begin{aligned}
\left\|v_{n}-S_{n} v_{n}\right\| & =\left\|v_{n}-w_{n}+w_{n}-S_{n} w_{n}+S_{n} w_{n}-S_{n} v_{n}\right\| \\
& \leq\left\|v_{n}-w_{n}\right\|+\left\|w_{n}-S_{n} w_{n}\right\|+\left\|w_{n}-v_{n}\right\|
\end{aligned}
$$

Since $\left\|w_{n}-v_{n}\right\| \rightarrow 0,\left\|w_{n}-S_{n} w_{n}\right\| \rightarrow 0$, we get $\left\|v_{n}-S_{n} v_{n}\right\| \rightarrow 0$.

Moreover, we note that

$$
\begin{aligned}
\left\|S v_{n}-v_{n}\right\| & \leq\left\|S v_{n}-S_{n} v_{n}\right\|+\left\|S_{n} v_{n}-v_{n}\right\| \\
& \leq \sup \left\{\left\|S w-S_{n} w\right\|: w \in\left\{v_{n}\right\}\right\}+\left\|S_{n} v_{n}-v_{n}\right\|
\end{aligned}
$$

By Lemma 2.3, we have $\left\|S v_{n}-v_{n}\right\| \rightarrow 0$.

Now, we prove that $\widetilde{w} \in \Omega$.

Since $\left\{v_{n}\right\}$ is bounded, we may assume that there exists a subsequence $\left\{v_{n_{i}}\right\}$ of $\left\{v_{n}\right\}$ which converges weakly to a point $\widetilde{w}$, i.e. $v_{n_{i}} \rightarrow \widetilde{w}$ as $i \rightarrow \infty$. Suppose that $\widetilde{w} \notin \operatorname{Fix}(S)$, since $v_{n_{i}} \rightarrow$ $\widetilde{w}$ and $S \widetilde{w} \neq \widetilde{w}$. Applying Opial's property, we obtain

$$
\begin{aligned}
\liminf _{i \rightarrow \infty}\left\|v_{n_{i}}-\widetilde{w}\right\| & <\liminf _{i \rightarrow \infty}\left\|v_{n_{i}}-S \widetilde{w}\right\| \\
& \leq \liminf _{i \rightarrow \infty}\left\{\left\|v_{n_{i}}-S v_{n_{i}}\right\|+\left\|S v_{n_{i}}-S \widetilde{w}\right\|\right\} \\
& \leq \liminf _{i \rightarrow \infty}\left\|v_{n_{i}}-\widetilde{w}\right\| .
\end{aligned}
$$

This is a contraction, then $\widetilde{w} \in \operatorname{Fix}(S)=\bigcap_{i=1}^{\infty} \operatorname{Fix}\left(S_{n}\right)$.

Since $\left\{w_{n}\right\}$ and $\left\{v_{n}\right\}$ are bounded, $\left\|w_{n}-v_{n}\right\| \rightarrow 0,\left\{w_{n}\right\}$ and $\left\{v_{n}\right\}$ have the same asymptotical behavior, we may assume that there exists a subsequence $\left\{w_{n_{i}}\right\}$ of $\left\{w_{n}\right\}$ which also converges weakly to the point $\widetilde{w}$, i.e. $w_{n_{i}} \rightarrow \widetilde{w}$ as $n_{i} \rightarrow \infty$. Suppose that $\widetilde{w} \neq J_{u_{n}}^{(U, K)}\left(I-\gamma G^{*} G\right) \widetilde{w}$, 
Applying Opial's property, we have

$$
\begin{aligned}
& \liminf _{i \rightarrow \infty}\left\|w_{n_{i}}-\widetilde{w}\right\| \\
& \quad<\liminf _{i \rightarrow \infty}\left\|w_{n_{i}}-J_{u_{n}}^{(U, K)}\left(I-\gamma G^{*} G\right) \widetilde{w}\right\| \\
& \leq \liminf _{i \rightarrow \infty}\left\{\left\|w_{n_{i}}-J_{u_{n}}^{(U, K)}\left(I-\gamma G^{*} G\right) w_{n_{i}}\right\|\right. \\
& \left.\quad+\left\|J_{u_{n}}^{(U, K)}\left(I-\gamma G^{*} G\right) w_{n_{i}}-J_{u_{n}}^{(U, K)}\left(I-\gamma G^{*} G\right) \widetilde{w}\right\|\right\} \\
& \leq \liminf _{i \rightarrow \infty}\left\{\left\|w_{n_{i}}-v_{n_{i}}\right\|+\left\|w_{n_{i}}-\widetilde{w}\right\|\right\} \\
& \leq \liminf _{i \rightarrow \infty}\left\|w_{n_{i}}-\widetilde{w}\right\| .
\end{aligned}
$$

This is a contraction, then $\widetilde{w}=J_{u_{n}}^{(U, K)}\left(I-\gamma G^{*} G\right) \widetilde{w}$, by Lemma 3.1 we have $\widetilde{w} \in \Gamma$. Thus, $\widetilde{w}$ is a solution of SEVIP, i.e. $\widetilde{w} \in \Omega=\operatorname{Fix}(S) \cap \Gamma$.

We now show that $\limsup _{n \rightarrow \infty}\left\langle\sigma f\left(w^{*}\right)-D w^{*}, w_{n}-w^{*}\right\rangle \leq 0$, where $w^{*}=P_{\Omega}(I-D+$ $\sigma f)\left(w^{*}\right)$ is the unique solution of VI (13).

Indeed, we can choose a subsequence $\left\{w_{n_{i}}\right\}$ of $\left\{w_{n}\right\}$ such that

$$
\limsup _{n \rightarrow \infty}\left\langle\sigma f\left(w^{*}\right)-D w^{*}, w_{n}-w^{*}\right\rangle=\lim _{n \rightarrow \infty}\left\langle\sigma f\left(w^{*}\right)-D w^{*}, w_{n_{i}}-w^{*}\right\rangle .
$$

We also assume that $w_{n_{i}} \rightarrow \widetilde{w}$. Therefore

$$
\begin{aligned}
& \limsup _{n \rightarrow \infty}\left\langle\sigma f\left(w^{*}\right)-D w^{*}, w_{n}-w^{*}\right\rangle \\
& \quad=\lim _{n_{i} \rightarrow \infty}\left\langle\sigma f\left(w^{*}\right)-D w^{*}, w_{n_{i}}-w^{*}\right\rangle \\
& \quad=\left\langle\sigma f\left(w^{*}\right)-D w^{*}, \widetilde{w}-w^{*}\right\rangle \\
& \quad=\left\langle(I-D+\sigma f) w^{*}-w^{*}, \widetilde{w}-w^{*}\right\rangle \\
& \quad=\left\langle(I-D+\sigma f) w^{*}-P_{\Omega}(I-D+\sigma f) w^{*}, \widetilde{w}-P_{\Omega}(I-D+\sigma f) w^{*}\right\rangle \\
& \quad \leq 0 .
\end{aligned}
$$

Then

$$
\limsup _{n \rightarrow \infty}\left\langle\sigma f\left(w^{*}\right)-D w^{*}, w_{n}-w^{*}\right\rangle \leq 0
$$

On the other hand, we will prove that $w^{*}=P_{\Omega}(I-D+\sigma f)\left(w^{*}\right)$ is the unique solution of VI (13).

Suppose $w^{*} \in \Omega$ and $w^{* *} \in \Omega$ both are solutions to VI (13), then

$$
\left\langle\sigma f\left(w^{*}\right)-D w^{*}, w^{* *}-w^{*}\right\rangle \leq 0
$$

and

$$
\left\langle\sigma f\left(w^{* *}\right)-D w^{* *}, w^{*}-w^{* *}\right\rangle \leq 0
$$


From the above inequalities we have

$$
\left\langle(D-\sigma f) w^{* *}-(D-\sigma f) w^{*}, w^{* *}-w^{*}\right\rangle \leq 0 .
$$

By Lemma 2.2, we have $D-\sigma f$ is strongly monotone, then $w^{* *}=w^{*}$, the uniqueness is proved.

Finally, we show that $w_{n}$ converges strongly to $w^{*}$ as $n \rightarrow \infty$.

$$
\begin{aligned}
\| w_{n} & -w^{*} \|^{2} \\
= & \left\|\alpha_{n} \sigma f\left(w_{n}\right)+\left(I-\alpha_{n} D\right) S_{n} v_{n}-w^{*}\right\|^{2} \\
= & \left\|\left(I-\alpha_{n} D\right)\left(S_{n} v_{n}-w^{*}\right)+\alpha_{n}\left(\sigma f\left(w_{n}\right)-D w^{*}\right)\right\|^{2} \\
\leq & \left\|\left(I-\alpha_{n} D\right)\left(S_{n} v_{n}-w^{*}\right)\right\|^{2}+2 \alpha_{n}\left\langle\sigma f\left(w_{n}\right)-D w^{*}, w_{n}-w^{*}\right\rangle \\
\leq & \left(1-\alpha_{n} \bar{\gamma}\right)^{2}\left\|v_{n}-w^{*}\right\|^{2}+2 \alpha_{n} \sigma\left\langle f\left(w_{n}\right)-f\left(w^{*}\right), w_{n}-w^{*}\right\rangle \\
& +2 \alpha_{n}\left\langle\sigma f\left(w^{*}\right)-D w^{*}, w_{n}-w^{*}\right\rangle \\
\leq & \left(1-\alpha_{n} \bar{\gamma}\right)^{2}\left\|w_{n}-w^{*}\right\|^{2}+2 \alpha_{n} \sigma k\left\|w_{n}-w^{*}\right\|^{2}+2 \alpha_{n}\left\langle\sigma f\left(w^{*}\right)-D w^{*}, w_{n}-w^{*}\right\rangle .
\end{aligned}
$$

This implies that

$$
2(\bar{\gamma}-\sigma k)\left\|w_{n}-w^{*}\right\|^{2} \leq \alpha_{n} \bar{\gamma}^{2}\left\|w_{n}-w^{*}\right\|^{2}+2\left\langle\sigma f\left(w^{*}\right)-D w^{*}, w_{n}-w^{*}\right\rangle
$$

From condition (i) and (20), we can obtain the desired conclusion

$$
\lim _{n \rightarrow \infty}\left\|w_{n}-w^{*}\right\|=0
$$

This completes the proof.

Theorem 3.2 Let $H_{1}, H_{2}, H_{3}, A, B, A^{*}, B^{*}, U, K, J_{u_{n}}^{(U, K)}, G, G^{*}, f, S_{n}, D, S$ be the same as them of Theorem 3.1. Let $w_{n}$ be generated by

$$
\left\{\begin{array}{l}
v_{n}=J_{u_{n}}^{(U, K)}\left(I-\gamma G^{*} G\right) w_{n} \\
w_{n+1}=\alpha_{n} \sigma f\left(w_{n}\right)+\left(I-\alpha_{n} D\right) S_{n} v_{n},
\end{array}\right.
$$

suppose $S_{n}$ satisfies the AKTT condition, $\operatorname{Fix}(S)=\bigcap_{i=1}^{\infty} \operatorname{Fix}\left(S_{n}\right)$. If the solution set $\Omega=$ $\operatorname{Fix}(S) \cap \Gamma$ is nonempty and the following conditions are satisfied:

(i) $\alpha_{n} \in(0,1), \lim _{n \rightarrow \infty} \alpha_{n}=0, \sum_{n=0}^{\infty} \alpha_{n}=\infty$;

(ii) $\sum_{n=0}^{\infty}\left|\alpha_{n+1}-\alpha_{n}\right|<\infty$;

(iii) $\sum_{n=0}^{\infty}\left|u_{n+1}-u_{n}\right|<\infty$;

(iv) $0<\bar{\gamma}<\frac{1}{\alpha_{n}}, 0<\sigma<\frac{\bar{\gamma}}{k}$,

then the sequence $w_{n}$ converges strongly to a point $w^{*}$, where $w^{*}=P_{\Omega}(I-D-\sigma f)\left(w^{*}\right)$ is a unique solution of the variational inequalities

$$
\left\langle(D-\sigma f) w^{*}, w^{*}-z\right\rangle \leq 0, \quad z \in \Omega .
$$


Proof We first prove the $w_{n}$ is bounded.

For any given $z \in \Omega$, we have $z=J_{u_{i}}^{\left(U_{i}, K_{i}\right)}\left(I-\gamma G^{*} G\right) z$ and $z \in F(S)=\bigcap_{n=1}^{\infty} F\left(S_{n}\right)$. By Lemma 2.1 and Proposition 3.1, we have

$$
\begin{aligned}
\left\|w_{n+1}-z\right\| & =\left\|\alpha_{n} \sigma f\left(w_{n}\right)+\left(I-\alpha_{n} D\right) S_{n} v_{n}-z\right\| \\
& =\left\|\alpha_{n} \sigma f\left(w_{n}\right)-\alpha_{n} D z+\left(I-\alpha_{n} D\right) S_{n} v_{n}-\left(I-\alpha_{n} D\right) S_{n} z\right\| \\
& \leq \alpha_{n}\left\|\sigma f\left(w_{n}\right)-D z\right\|+\left\|I-\alpha_{n} D\right\|\left\|v_{n}-z\right\| \\
& \leq \alpha_{n}\left\|\sigma f\left(w_{n}\right)-D z\right\|+\left(1-\alpha_{n} \bar{\gamma}\right)\left\|J_{u_{i}}^{\left(U_{i}, K_{i}\right)}\left(I-\gamma G^{*} G\right) w_{n}-z\right\| \\
& \leq \alpha_{n}\left\|\sigma f\left(w_{n}\right)-D z\right\|+\left(1-\alpha_{n} \bar{\gamma}\right)\left\|w_{n}-z\right\| \\
& \leq \alpha_{n}\left\|\sigma\left(f\left(w_{n}\right)-f(z)\right)+(\sigma f(z)-D z)\right\|+\left(1-\alpha_{n} \bar{\gamma}\right)\left\|w_{n}-z\right\| \\
& \leq \alpha_{n} \sigma k\left\|w_{n}-z\right\|+\alpha_{n}\|\sigma f(z)-D z\|+\left(1-\alpha_{n} \bar{\gamma}\right)\left\|w_{n}-z\right\| \\
& =\left(I-\alpha_{n}(\bar{\gamma}-\sigma k)\right)\left\|w_{n}-z\right\|+\alpha_{n}(\bar{\gamma}-\sigma k) \frac{\|\sigma f(z)-D z\|}{\bar{\gamma}-\sigma k} \\
& \leq \max \left\{\left\|w_{n}-z\right\|, \frac{\|\sigma f(z)-D z\|}{\bar{\gamma}-\sigma k}\right\} .
\end{aligned}
$$

By a simple induction, we have

$$
\left\|w_{n}-z\right\| \leq \max \left\{\left\|w_{0}-z\right\|, \frac{\|\sigma f(z)-D z\|}{\bar{\gamma}-\sigma k}\right\}
$$

Therefore, $\left\{w_{n}\right\}$ is bounded, and so are $\left\{v_{n}\right\},\left\{f\left(w_{n}\right)\right\},\left\{S_{n} v_{n}\right\}$.

From (17), by a similar argument to the proof of Theorem 3.1, we derive that

$$
\begin{aligned}
& \left\|w_{n+1}-z\right\|^{2} \\
& =\left\|\alpha_{n} \sigma f\left(w_{n}\right)+\left(I-\alpha_{n} D\right) S_{n} v_{n}-z\right\|^{2} \\
& =\left\|\left(I-\alpha_{n} D\right)\left(S_{n} v_{n}-z\right)+\alpha_{n}\left(\sigma f\left(w_{n}\right)-D z\right)\right\|^{2} \\
& \leq\left\|\left(I-\alpha_{n} D\right)\left(S_{n} v_{n}-z\right)\right\|^{2}+2 \alpha_{n}\left\langle\sigma f\left(w_{n}\right)-D z, w_{n+1}-z\right\rangle \\
& \leq\left(1-\alpha_{n} \bar{\gamma}\right)^{2}\left\|v_{n}-z\right\|^{2}+2 \alpha_{n}\left\langle\sigma f\left(w_{n}\right)-D z, w_{n+1}-z\right\rangle \\
& \leq\left(1-\alpha_{n} \bar{\gamma}\right)\left\|w_{n}-z\right\|^{2}-\left(1-\alpha_{n} \bar{\gamma}\right) \gamma(2-\gamma L)\left\|G w_{n}\right\|^{2} \\
& \quad+2 \alpha_{n}\left\|\sigma f\left(w_{n}\right)-D z\right\|\left\|w_{n+1}-z\right\| .
\end{aligned}
$$

This implies that

$$
\begin{aligned}
& \left(1-\alpha_{n} \bar{\gamma}\right) \gamma(2-\gamma L)\left\|G w_{n}\right\|^{2} \\
& \quad \leq\left\|w_{n}-z\right\|^{2}-\left\|w_{n+1}-z\right\|^{2}+2 \alpha_{n}\left\|\sigma f\left(w_{n}\right)-D z\right\|\left\|w_{n}-z\right\| .
\end{aligned}
$$

Now, the rest of the proofs will be analyzed as two cases due to the monotone property of $\left\{\left\|w_{n}-z\right\|\right\}$.

Case 1: $\left\{\left\|w_{n}-z\right\|\right\}$ is a monotone sequence. 
Since $\left\{\left\|w_{n}-z\right\|\right\}$ is bounded, $\left\{\left\|w_{n}-z\right\|\right\}$ is convergent. Take the limit on both sides for (24), in view of condition (i). We have

$$
\left\|G w_{n}\right\| \rightarrow 0
$$

By the same argument as in the proof of Theorem 3.1, we derive that

$$
\left\|v_{n}-z\right\|^{2} \leq\left\|w_{n}-z\right\|^{2}-\left\|w_{n}-v_{n}\right\|^{2}+2 \gamma \sqrt{L}\left\|w_{n}-v_{n}\right\|\left\|G w_{n}\right\| .
$$

Then, from (23), (25), and (10), we derive that

$$
\begin{aligned}
\left\|w_{n+1}-z\right\|^{2} \leq & \left(1-\alpha_{n} \bar{\gamma}\right)^{2}\left\|v_{n}-z\right\|^{2}+2 \alpha_{n}\left\langle\sigma f\left(w_{n}\right)-D z, w_{n+1}-z\right\rangle \\
\leq & \left(1-\alpha_{n} \bar{\gamma}\right)\left\|w_{n}-z\right\|^{2}-\left(1-\alpha_{n} \bar{\gamma}\right)\left\|w_{n}-v_{n}\right\|^{2} \\
& +2\left(1-\alpha_{n} \bar{\gamma}\right) \gamma \sqrt{L}\left\|w_{n}-v_{n}\right\|\left\|G w_{n}\right\| \\
& +2 \alpha_{n}\left\|\sigma f\left(w_{n}\right)-D z\right\|\left\|w_{n+1}-z\right\| .
\end{aligned}
$$

Hence, we obtain

$$
\begin{gathered}
\left(1-\alpha_{n} \bar{\gamma}\right)\left\|w_{n}-v_{n}\right\|^{2} \leq \\
+w_{n}-z\left\|^{2}-\right\| w_{n+1}-z\left\|^{2}+2\left(1-\alpha_{n} \bar{\gamma}\right) \gamma \sqrt{L}\right\| w_{n}-v_{n}\|\| G w_{n} \| \\
+2 \alpha_{n}\left\|\sigma f\left(w_{n}\right)-D z\right\|\left\|w_{n}-z\right\| .
\end{gathered}
$$

Since $\left\{w_{n}\right\},\left\{v_{n}\right\},\left\{f\left(w_{n}\right)\right\}$ are bounded, $\lim _{n \rightarrow \infty}\left\{\left\|w_{n}-z\right\|\right\}$ exists and $\alpha_{n} \rightarrow 0$, then $\| w_{n}-$ $v_{n} \| \rightarrow 0$.

Indeed, $J_{u_{n}}^{(U, K)}\left(I-\gamma G^{*} G\right)$ is nonexpansive and by Lemma 2.5(iii) we derive that

$$
\begin{aligned}
\left\|v_{n+1}-v_{n}\right\|= & \left\|J_{u_{n+1}}^{(U, K)}\left(I-\gamma G^{*} G\right) w_{n+1}-J_{u_{n}}^{(U, K)}\left(I-\gamma G^{*} G\right) w_{n}\right\| \\
\leq & \left\|J_{u_{n+1}}^{(U, K)}\left(I-\gamma G^{*} G\right) w_{n+1}-J_{u_{n+1}}^{(U, K)}\left(I-\gamma G^{*} G\right) w_{n}\right\| \\
& \left.\quad+\left\|J_{u_{n+1}(U, K)}\right\| I-\gamma G^{*} G\right) w_{n}-J_{u_{n}}^{(U, K)}\left(I-\gamma G^{*} G\right) w_{n} \| \\
\leq & \left\|w_{n+1}-w_{n}\right\|+\frac{\left|u_{n+1}-u_{n}\right|}{u_{n+1}}\left\|J_{u_{n+1}}^{(U, K)}\left(I-\gamma G^{*} G\right) w_{n}-w_{n}\right\|
\end{aligned}
$$

Since $\liminf _{n \rightarrow \infty} u_{n}>0$, we may assume that there exists a real number $m$ such that $u_{n} \geq m>0$ for all $n \in N$. Then we have

$$
\begin{aligned}
\left\|v_{n+1}-v_{n}\right\| & \leq\left\|w_{n+1}-w_{n}\right\|+\frac{\left|u_{n+1}-u_{n}\right|}{m}\left\|J_{u_{n+1}}^{(u, K)}\left(I-\gamma G^{*} G\right) w_{n}-w_{n}\right\| \\
& \leq\left\|w_{n+1}-w_{n}\right\|+M_{1}\left|u_{n+1}-u_{n}\right|
\end{aligned}
$$

where $M_{1}=\sup \left\{\frac{1}{m}\left\|J_{u_{n+1}}^{(U, K)}\left(I-\gamma G^{*} G\right) w_{n}-w_{n}\right\|: n \in N\right\}$.

Thus, we get

$$
\begin{aligned}
& \left\|w_{n+2}-w_{n+1}\right\| \\
& \quad=\left\|\alpha_{n+1} \sigma f\left(w_{n+1}\right)+\left(I-\alpha_{n+1} D\right) S_{n+1} v_{n+1}-\alpha_{n} \sigma f\left(w_{n}\right)-\left(I-\alpha_{n} D\right) S_{n} v_{n}\right\|
\end{aligned}
$$




$$
\begin{aligned}
= & \| \alpha_{n+1} \sigma f\left(w_{n+1}\right)+\left(I-\alpha_{n+1} D\right) S_{n+1} v_{n+1}-\alpha_{n} \sigma f\left(w_{n}\right)-\left(I-\alpha_{n} D\right) S_{n} v_{n} \\
& -\left(I-\alpha_{n+1} D\right) S_{n+1} v_{n}+\left(I-\alpha_{n+1} D\right) S_{n+1} v_{n}-\left(I-\alpha_{n} D\right) S_{n+1} v_{n} \\
& +\left(I-\alpha_{n} D\right) S_{n+1} v_{n}-\alpha_{n+1} \sigma f\left(w_{n}\right)+\alpha_{n+1} \sigma f\left(w_{n}\right) \| \\
= & \|\left(I-\alpha_{n+1} D\right)\left(S_{n+1} v_{n+1}-S_{n+1} v_{n}\right)+\left(\alpha_{n}-\alpha_{n+1}\right) D S_{n+1} v_{n} \\
& +\left(I-\alpha_{n} D\right)\left(S_{n+1} v_{n}-S_{n} v_{n}\right)+\left(\alpha_{n+1}-\alpha_{n}\right) \sigma f\left(w_{n}\right)+\alpha_{n+1} \sigma\left(f\left(w_{n+1}\right)-f\left(w_{n}\right)\right) \| \\
\leq & \left(I-\alpha_{n+1} \bar{\gamma}\right)\left\|v_{n+1}-v_{n}\right\|+\left|\alpha_{n}-\alpha_{n+1}\right|\left\|D S_{n+1} v_{n}\right\|+\left(I-\alpha_{n} \bar{\gamma}\right)\left\|S_{n+1} v_{n}-S_{n} v_{n}\right\| \\
& +\left|\alpha_{n}-\alpha_{n+1}\right|\left\|\sigma f\left(w_{n}\right)\right\|+\alpha_{n+1} \sigma k\left\|w_{n+1}-w_{n}\right\| \\
= & \left(I-\alpha_{n+1} \bar{\gamma}\right)\left\|v_{n+1}-v_{n}\right\|+\alpha_{n+1} \sigma k\left\|w_{n+1}-w_{n}\right\| \\
& +\left|\alpha_{n}-\alpha_{n+1}\right|\left(\left\|D S_{n+1} v_{n}\right\|+\left\|\sigma f\left(w_{n}\right)\right\|\right)+\left(I-\alpha_{n} \bar{\gamma}\right)\left\|S_{n+1} v_{n}-S_{n} v_{n}\right\| \\
\leq & \left(1-\alpha_{n+1}(\bar{\gamma}-\sigma k)\right)\left\|w_{n+1}-w_{n}\right\|+M_{1}\left|u_{n+1}-u_{n}\right| \\
& +\left|\alpha_{n}-\alpha_{n+1}\right|\left(\left\|D S_{n+1} v_{n}\right\|+\left\|\sigma f\left(w_{n}\right)\right\|\right)+\left\|S_{n+1} v_{n}-S_{n} v_{n}\right\| \\
\leq & \left(1-\alpha_{n+1}(\bar{\gamma}-\sigma k)\right)\left\|w_{n+1}-w_{n}\right\|+M_{1}\left|u_{n+1}-u_{n}\right|+2 M_{2}\left|\alpha_{n}-\alpha_{n+1}\right|+L_{n} \\
\leq & \left(1-\alpha_{n+1}(\bar{\gamma}-\sigma k)\right)\left\|w_{n+1}-w_{n}\right\|+M_{3}\left(\left|u_{n+1}-u_{n}\right|+\left|\alpha_{n}-\alpha_{n+1}\right|\right)+L_{n},
\end{aligned}
$$

where $M_{2}=\max \left\{\sup _{n \in N}\left\|D S_{n+1} v_{n}\right\|, \sup _{n \in N}\left\|\sigma f\left(w_{n}\right)\right\|\right\}, \quad M_{3}=\max \left\{M_{1}, 2 M_{2}\right\}, \quad L_{n}=$ $\sup \left\{\left\|S_{n+1} v-S_{n} v\right\|: v \in v_{n}\right\}$.

By Lemma 2.4, we have $\left\|w_{n+1}-w_{n}\right\| \rightarrow 0$.

Then, from condition (i) and $\left|u_{n+1}-u_{n}\right| \rightarrow 0$, we have $\left\|v_{n+1}-v_{n}\right\| \rightarrow 0$.

Indeed,

$$
\begin{aligned}
\left\|w_{n}-S_{n} v_{n}\right\| \leq & \left\|w_{n}-S_{n-1} v_{n-1}\right\|+\left\|S_{n-1} v_{n-1}-S_{n-1} v_{n}\right\|+\left\|S_{n-1} v_{n}-S_{n} v_{n}\right\| \\
\leq & \alpha_{n-1}\left\|\sigma f\left(w_{n-1}\right)-D S_{n-1} v_{n-1}\right\|+\left\|v_{n-1}-v_{n}\right\| \\
& +\sup \left\{\left\|S_{n-1} w-S_{n} w\right\|: w \in v_{n}\right\} .
\end{aligned}
$$

Since $\left\|v_{n+1}-v_{n}\right\| \rightarrow 0$, by Lemma 2.3 and condition (i), we have $\left\|w_{n}-S_{n} v_{n}\right\| \rightarrow 0$.

Then

$$
\left\|S_{n} v_{n}-v_{n}\right\| \leq\left\|S_{n} v_{n}-w_{n}\right\|+\left\|w_{n}-v_{n}\right\|
$$

Since $\left\|w_{n}-S_{n} v_{n}\right\| \rightarrow 0$ and $\left\|w_{n}-v_{n}\right\| \rightarrow 0$, we get $\left\|S_{n} v_{n}-v_{n}\right\| \rightarrow 0$.

Moreover, we note that

$$
\begin{aligned}
\left\|S v_{n}-v_{n}\right\| & \leq\left\|S v_{n}-S_{n} v_{n}\right\|+\left\|S_{n} v_{n}-v_{n}\right\| \\
& \leq \sup \left\{\left\|S w-S_{n} w\right\|: w \in\left\{v_{n}\right\}\right\}+\left\|S_{n} v_{n}-v_{n}\right\| .
\end{aligned}
$$

By Lemma 2.3 we have

$$
\left\|S v_{n}-v_{n}\right\| \rightarrow 0
$$


Since $\left\{v_{n}\right\}$ is bounded, we may assume that there exists a subsequence $\left\{v_{n_{i}}\right\}$ of $\left\{v_{n}\right\}$ which converges weakly to a point $\widetilde{w}$, i.e. $v_{n_{i}} \rightarrow \widetilde{w}$ as $i \rightarrow \infty,\left\{w_{n}\right\}$ and $\left\{v_{n}\right\}$ are bounded, $\left\{w_{n}\right\}$ and $\left\{v_{n}\right\}$ have the same asymptotical behavior.

We may assume that there exists a subsequence $\left\{w_{n_{i}}\right\}$ of $\left\{w_{n}\right\}$ which also converges weakly to the point $\widetilde{w}$, i.e. $w_{n_{i}} \rightarrow \widetilde{w}$ as $i \rightarrow \infty$. By the same argument as in the proof of Theorem 3.1, we derive that $\widetilde{w}$ is a solution of SEVIP, i.e. $\widetilde{w} \in \Omega=\operatorname{Fix}(S) \cap \Gamma$.

Next, by the same argument as in the proof of Theorem 3.1, we derive that

$$
\limsup _{n \rightarrow \infty}\left\langle\sigma f\left(w^{*}\right)-D w^{*}, w_{n}-w^{*}\right\rangle \leq 0
$$

where $w^{*}=P_{\Omega}(I-D+\sigma f)\left(w^{*}\right)$ is the unique solution of VI (22).

Finally, we show that $w_{n}$ converges strongly to $w^{*}$ as $n \rightarrow \infty$.

$$
\begin{aligned}
&\left\|w_{n+1}-w^{*}\right\|^{2} \\
&=\left\|\alpha_{n} \sigma f\left(w_{n}\right)+\left(I-\alpha_{n} D\right) S_{n} v_{n}-w^{*}\right\|^{2} \\
&=\left\|\left(I-\alpha_{n} D\right)\left(S_{n} v_{n}-w^{*}\right)+\alpha_{n}\left(\sigma f\left(w_{n}\right)-D w^{*}\right)\right\|^{2} \\
& \leq\left\|\left(I-\alpha_{n} D\right)\left(S_{n} v_{n}-w^{*}\right)\right\|^{2}+2 \alpha_{n}\left\langle\sigma f\left(w_{n}\right)-D w^{*}, w_{n+1}-w^{*}\right\rangle \\
& \leq\left(1-\alpha_{n} \bar{\gamma}\right)^{2}\left\|v_{n}-w^{*}\right\|^{2}+2 \alpha_{n} \sigma\left\langle f\left(w_{n}\right)-f\left(w^{*}\right), w_{n+1}-w^{*}\right\rangle \\
&+2 \alpha_{n}\left\langle\sigma f\left(w^{*}\right)-D w^{*}, w_{n+1}-w^{*}\right\rangle \\
& \leq\left(1-\alpha_{n} \bar{\gamma}\right)^{2}\left\|w_{n}-w^{*}\right\|^{2}+2 \alpha_{n} \sigma k\left\|w_{n}-w^{*}\right\|\left\|w_{n+1}-w^{*}\right\| \\
&+2 \alpha_{n}\left\langle\sigma f\left(w^{*}\right)-D w^{*}, w_{n+1}-w^{*}\right\rangle \\
& \leq\left(1-\alpha_{n} \bar{\gamma}\right)^{2}\left\|w_{n}-w^{*}\right\|^{2}+\alpha_{n} \sigma k\left(\left\|w_{n}-w^{*}\right\|^{2}+\left\|w_{n+1}-w^{*}\right\|^{2}\right) \\
&+2 \alpha_{n}\left\langle\sigma f\left(w^{*}\right)-D w^{*}, w_{n+1}-w^{*}\right\rangle .
\end{aligned}
$$

It follows that

$$
\begin{aligned}
\left\|w_{n+1}-w^{*}\right\|^{2} \leq & \frac{1-2 \alpha_{n} \bar{\gamma}+\alpha_{n}^{2} \bar{\gamma}^{2}+\alpha_{n} \sigma k}{1-\alpha_{n} \sigma k}\left\|w_{n}-w^{*}\right\|^{2} \\
& +\frac{2 \alpha_{n}}{1-\alpha_{n} \sigma k}\left\langle\sigma f\left(w^{*}\right)-D w^{*}, w_{n+1}-w^{*}\right\rangle \\
= & \left(1-\frac{2 \alpha_{n}(\bar{\gamma}-\sigma k)}{1-\alpha_{n} \sigma k}\right)\left\|w_{n}-w^{*}\right\|^{2}+\frac{\alpha_{n}^{2} \bar{\gamma}^{2}}{1-\alpha_{n} \sigma k}\left\|w_{n}-w^{*}\right\|^{2} \\
& +\frac{2 \alpha_{n}}{1-\alpha_{n} \sigma k}\left\langle\sigma f\left(w^{*}\right)-D w^{*}, w_{n+1}-w^{*}\right\rangle,
\end{aligned}
$$

where $\gamma_{n}=\frac{2 \alpha_{n}(\bar{\gamma}-\sigma k)}{1-\alpha_{n} \sigma k}$ and $\delta_{n}=\frac{\alpha_{n}^{2} \bar{\gamma}^{2}}{1-\alpha_{n} \sigma k}\left\|w_{n}-w^{*}\right\|^{2}+\frac{2 \alpha_{n}}{1-\alpha_{n} \sigma k}\left\langle\sigma f\left(w^{*}\right)-D w^{*}, w_{n+1}-w^{*}\right\rangle$.

Hence, all conditions of Lemma 2.4 are satisfied.

Therefore, we immediately deduce that $w_{n} \rightarrow w^{*}$.

Case 2: The sequence $\left\{\left\|w_{n}-z\right\|\right\}$ is not monotone.

By Lemma 2.6, there exists a sequence of positive integers: $\{\tau(n)\}, n \geq n_{0}$, where $n_{0}$ is large enough such that

$$
\tau(n)=\max \left\{k \leq n:\left\|w_{k}-w^{*}\right\| \leq\left\|w_{k+1}-w^{*}\right\|\right\} .
$$


It is easy to see that $\{\tau(n)\}$ is nondecreasing and $\tau(n) \rightarrow \infty$ as $n \rightarrow \infty$.

We have $\left\|w_{\tau(n)}-w^{*}\right\|<\left\|w_{\tau(n)+1}-w^{*}\right\| ;\left\|w_{n}-w^{*}\right\|<\left\|w_{\tau(n)+1}-w^{*}\right\|$.

Just as the argument of Case 1, we have

$$
\left\{\begin{array}{l}
\lim _{n \rightarrow \infty}\left\|w_{\tau(n)}-v_{\tau(n)}\right\|=0 \\
\lim _{n \rightarrow \infty}\left\|v_{\tau(n)+1}-v_{\tau(n)}\right\|=\lim _{n \rightarrow \infty}\left\|w_{\tau(n)+1}-w_{\tau(n)}\right\|=0 \\
\lim _{n \rightarrow \infty}\left\|S v_{\tau(n)}-v_{\tau(n)}\right\|=0 \\
\limsup _{n \rightarrow \infty}\left\langle\sigma f\left(w^{*}\right)-D w^{*}, w_{\tau(n)}-w^{*}\right\rangle \leq 0 .
\end{array}\right.
$$

According to Case 1, we have $\lim _{n \rightarrow \infty}\left\|w_{\tau(n)}-w^{*}\right\|=0$ and $\lim _{n \rightarrow \infty}\left\|w_{\tau(n)+1}-w^{*}\right\|=0$.

Finally, from Lemma 2.6, we get

$$
0 \leq\left\|w_{n}-w^{*}\right\| \leq \max \left\{\left\|w_{n}-w^{*}\right\|,\left\|w_{\tau(n)}-w^{*}\right\|\right\} \leq\left\|w_{\tau(n)+1}-w^{*}\right\| \rightarrow 0, \quad n \rightarrow \infty .
$$

Therefore, the sequence $\left\{w_{n}\right\}$ converges strongly to $w^{*}$.

This completes the proof.

Corollary 3.1 Let $H_{1}, H_{2}, H_{3}, A, B, A^{*}, B^{*}, U, K, J_{u_{n}}^{(U, K)}, G, G^{*}, f, S_{n}$, S be the same as them of Theorem 3.1. Let $w_{n}$ be generated by

$$
\left\{\begin{array}{l}
v_{n}=J_{u_{n}}^{(U, K)}\left(I-\gamma G^{*} G\right) w_{n} \\
w_{n+1}=\alpha_{n} f\left(w_{n}\right)+\left(1-\alpha_{n}\right) S_{n} v_{n} .
\end{array}\right.
$$

Suppose $S_{n}$ satisfies the AKTT condition, $\operatorname{Fix}(S)=\bigcap_{n=1}^{\infty} \operatorname{Fix}\left(S_{n}\right)$. If the solution set $\Omega=$ $\operatorname{Fix}(S) \cap \Gamma$ is nonempty and the following conditions are satisfied:

(i) $\alpha_{n} \in(0,1), \lim _{n \rightarrow \infty} \alpha_{n}=0, \sum_{n=0}^{\infty} \alpha_{n}=\infty$;

(ii) $\sum_{n=0}^{\infty}\left|\alpha_{n+1}-\alpha_{n}\right|<\infty$;

(iii) $\sum_{n=0}^{\infty}\left|u_{n+1}-u_{n}\right|<\infty$,

then the sequence $w_{n}$ converges strongly to $w^{*}$, where $w^{*}=P_{\Omega_{2}} f\left(w^{*}\right)$.

Corollary 3.2 Let $H_{1}, H_{2}, H_{3}, A, B, A^{*}, B^{*}, U, K, J_{u_{n}}^{(U, K)}, G, G^{*}, f, S_{n}, S$ be the same as them of Theorem 3.1. Let $\left\{\omega_{k}\right\}$ be a sequence of positive real numbers with $\sum_{k=1}^{\infty} \omega_{k}=1$, $S=\sum_{k=1}^{\infty} \omega_{k} S_{k}, L_{n}=\sum_{k=1}^{n} \frac{\omega_{k}}{M_{n}} S_{k}$, and $M_{n}=\sum_{k=1}^{n} \omega_{k}$. Let $w_{n}$ be generated by

$$
\left\{\begin{array}{l}
v_{n}=J_{u_{n}}^{(U, K)}\left(I-\gamma G^{*} G\right) w_{n} ; \\
w_{n+1}=\alpha_{n} f\left(w_{n}\right)+\left(1-\alpha_{n}\right) L_{n} v_{n} .
\end{array}\right.
$$

Suppose $S_{n}$ satisfies the AKTT condition, $\operatorname{Fix}(S)=\bigcap_{n=1}^{\infty} \operatorname{Fix}\left(S_{n}\right)$. If the solution set $\Omega=$ $\operatorname{Fix}(S) \cap \Gamma$ is nonempty and the following conditions are satisfied:

(i) $\alpha_{n} \in(0,1), \lim _{n \rightarrow \infty} \alpha_{n}=0, \sum_{n=0}^{\infty} \alpha_{n}=\infty$;

(ii) $\sum_{n=0}^{\infty}\left|\alpha_{n+1}-\alpha_{n}\right|<\infty$;

(iii) $\sum_{n=0}^{\infty}\left|u_{n+1}-u_{n}\right|<\infty$.

Then the sequence $w_{n}$ converges strongly to a point $w^{*}$, where $w^{*}=P_{\Omega} f\left(w^{*}\right)$.

It should be noted that by Bruck's lemma [16] and He-Guo's lemma [17] each $L_{n}$ is also nonexpansive mapping and $\operatorname{Fix}(S)=\bigcap_{n=1}^{\infty} \operatorname{Fix}\left(S_{n}\right)$. 
Table 1 Numerical results for some initial points $\left(x_{0}, y_{0}\right)=(0.01,0.01),(1,1),(15,15)$

\begin{tabular}{llll}
\hline Initial point & $\boldsymbol{\varepsilon}$ & Iter. & Time \\
\hline$(0.01,0.01)$ & 0.00001 & 2 & 0.00251 \\
$(0.01,0.01)$ & 0.00001 & 2 & 0.00268 \\
$(1,1)$ & 0.00001 & 5 & 0.00452 \\
$(1,1)$ & 0.00001 & 6 & 0.00550 \\
$(15,15)$ & 0.00001 & 8 & 0.00606 \\
$(15,15)$ & 0.00001 & 8 & 0.00790 \\
\hline
\end{tabular}

Table 2 Numerical results for some different $u_{n}=1,0.5,0.2,0.1$

\begin{tabular}{llrl}
\hline $\boldsymbol{u}_{\boldsymbol{n}}$ & $\boldsymbol{\varepsilon}$ & Iter. & Time \\
\hline 1 & 0.00001 & 22 & 0.02175 \\
1 & 0.00001 & 21 & 0.01469 \\
0.5 & 0.00001 & 36 & 0.02851 \\
0.5 & 0.00001 & 37 & 0.03045 \\
0.2 & 0.00001 & 125 & 0.11522 \\
0.2 & 0.00001 & 113 & 0.08003 \\
0.1 & 0.00001 & 1223 & 0.94707 \\
0.1 & 0.00001 & 757 & 0.53070 \\
\hline
\end{tabular}

\section{Numerical example}

In this section, we give an example and numerical results to illustrate our algorithms and the main result of this paper. All the experiment are performed on a personal Lenovo computer with Intel Core i3-2485M CPU 2.30 GHz and RAM 2.00 GB.

Example 4.1 Let $H_{1}=R^{4}, H_{2}=R^{4}, H_{3}=R^{4}$, two operators of matrix multiplication $U$ : $R^{4} \rightarrow R^{4}, K: R^{4} \rightarrow R^{4}$ defined by $U(x)=T_{1}(x), K(x)=T_{2}(x)$, where

$$
T_{1}=\left[\begin{array}{cccc}
8 & 0 & 0 & 0 \\
0 & 12 & 0 & 0 \\
0 & 0 & 7 & 0 \\
0 & 0 & 0 & 20
\end{array}\right], \quad T_{2}=\left[\begin{array}{cccc}
3 & 0 & 0 & 0 \\
0 & 6 & 0 & 0 \\
0 & 0 & 2 & 0 \\
0 & 0 & 0 & 12
\end{array}\right]
$$

put $S_{n}(x)=\frac{1}{1+2 n} x, \sigma=1, D=I$, then $U, K, S_{n}$ satisfy all conditions of Theorem 3.1 and Corollary 3.1. We know (12) is equivalent to the following step:

$$
\left\{\begin{array}{l}
x_{n+1}=\alpha_{n} f_{1}\left(x_{n}\right)+\left(1-\alpha_{n}\right) S_{n} J_{u_{n}}^{U}\left(x_{n}-\gamma A^{T}\left(A x_{n}-B y_{n}\right)\right) \\
y_{n+1}=\alpha_{n} f_{2}\left(y_{n}\right)+\left(1-\alpha_{n}\right) S_{n} J_{u_{n}}^{K}\left(y_{n}+\gamma B^{T}\left(A x_{n}-B y_{n}\right)\right) .
\end{array}\right.
$$

Note that if $T_{1}, T_{2}$ are positive linear operators, then they are maximal monotone. We defined the resolvent mappings $J_{u_{n}}^{U}=\left(I+u_{n} U\right)^{-1}, J_{u_{n}}^{K}=\left(I+u_{n} K\right)^{-1}$, where $u_{n}>0$. Then we present the following algorithm.

\section{Algorithm 4.1}

Step 0 . Choose initial point $\left(x_{0}, y_{0}\right) \in\left(0,1 \times 10^{5}\right) \times\left(0,1 \times 10^{5}\right), c>0, \gamma \in\left(0, \frac{2}{\lambda_{A}+\lambda_{A}}\right)$ arbitrarily and put $n=1$.

Step 1. Compute $\left(x_{n+1}, y_{n+1}\right)$ as follows:

$$
\left\{\begin{array}{l}
x_{n+1}=\alpha_{n} f_{1}\left(x_{n}\right)+\left(1-\alpha_{n}\right) S_{n} J_{u_{n}}^{U}\left(x_{n}-\gamma A^{T}\left(A x_{n}-B y_{n}\right)\right) \\
y_{n+1}=\alpha_{n} f_{2}\left(y_{n}\right)+\left(1-\alpha_{n}\right) S_{n} J_{u_{n}}^{K}\left(y_{n}+\gamma B^{T}\left(A x_{n}-B y_{n}\right)\right) .
\end{array}\right.
$$


Step 2. Set $\left\|A x_{n}-B y_{n}\right\|<\varepsilon$ as the stop criterion, else set $n=n+1$ and go to step 1 .

Table 1 shows the numerical results of Algorithm 4.1 with different initial points.

Table 2 shows that decreasing of $u_{n}$ has an effect on the number of iterations, that is, $u_{n}$ will converge faster to a solution when $u_{n}$ is increased.

Competing interests

The authors declare that they have no competing interests.

\section{Authors' contributions}

All authors contributed equally and significantly in writing this paper. All authors read and approved the final manuscript.

\section{Author details}

${ }^{1}$ Department of Mathematics, Tianjin Polytechnic University, Tianjin, 300387, China. ${ }^{2}$ School of Mathematics and Statistics, Xidian University, Xian, 710071, China.

\section{Acknowledgements}

We wish to thank the referees for their helpful comments and suggestions. This research was supported by NSFC Grant No. 11071279 and Tian Yuan Special Foundation (No. 11426167).

Received: 8 August 2015 Accepted: 16 November 2015 Published online: 01 December 2015

\section{References}

1. Moudafi, A: Alternating CQ-algorithm for convex feasibility and split fixed point problems. J. Nonlinear Convex Anal. 15(4), 809-818 (2014)

2. Censor, Y, Bortfeld, T, Martin, B, Trofimov, A: A unified approach for inversion problems in intensity-modulated therapy. Phys. Med. Biol. 51, 2353-2365 (2006)

3. Byrne, C: Iterative oblique projection onto convex sets and the split feasibility problem. Inverse Probl. 18, 441-453 (2002)

4. Byrne, C: A unified treatment of some iterative algorithms in signal processing and image reconstruction. Inverse Probl. 20(1), 103-120 (2004)

5. Moudafi, A: Split monotone variational inclusions. J. Optim. Theory Appl. 150, 275-283 (2011)

6. Byrne, C, Censor, Y, Gibali, A, Reich, S: Weak and strong convergence of algorithms for the split common null point problem. J. Nonlinear Convex Anal. 13, 759-775 (2012)

7. Moudafi, A: Viscosity approximation methods for fixed points problems. J. Math. Anal. Appl. 241, $46-55$ (2000)

8. Marino, G, Xu, HK: A general iterative method for nonexpansive mappings in Hilbert spaces. J. Math. Anal. Appl. 318, 43-52 (2006)

9. Kazmi, KR, Rizvi, SH: An iterative method for split variational inclusion problem and fixed point problem for a nonexpansive mapping. Optim. Lett. 8(3), 1113-1124 (2014)

10. Sitthithakerngkiet, K, Deepho, J, Kumam, P: A hybrid viscosity algorithm via modify the hybrid steepest descent method for solving the split variational inclusion in image reconstruction and fixed point problems. Appl. Math. Comput. 250, 986-1001 (2015)

11. Marino, G, Xu, HK: A general iterative method for nonexpansive mappings in Hilbert spaces. J. Math. Anal. Appl. 318(1), 43-52 (2006)

12. Aoyama, K, Kimura, Y, Takahashi, W, Toyoda, M: Approximation of common fixed points of a countable family of nonexpansive mappings in a Banach space. Nonlinear Anal., Theory Methods Appl. 67(8), 2350-2360 (2007)

13. Chang, S-S, Wang, L, Tang, YK, Wang, G: Moudafi's open question and simultaneous iterative algorithm for general split equality variational inclusion problems and general split equality optimization problems. Fixed Point Theory Appl. 2014, Article ID 215 (2014)

14. Takahashi, W: Nonlinear Functional Analysis. Fixed Point Theory and Its Application. Yokohama Publishers, Yokohama (2000)

15. Mainge, P-E: Strong convergence of projected subgradient methods for nonsmooth and nonstrictly convex minimization. Set-Valued Anal. 16(7-8), 899-912 (2008)

16. Bruck, R-E: Properties of fixed-point sets of nonexpansive mappings in Banach spaces. Trans. Am. Math. Soc. 179, 251-262 (1973)

17. He, SN, Guo, J: Iterative algorithm for common fixed points of infinite family of nonexpansive mappings in Banach spaces. J. Appl. Math. 2012, Article ID 787419 (2012) 